\title{
LA HISTORIA DE LA EDUCACIÓN Y LA INVESTIGACIÓN CIENTÍFICA EN BRASIL
}

\section{ARTÍ́CULO ORIGINAL}

FREITAS, Roberto Araujo de Moraes ${ }^{1}$

MARTINS, Priscila Bernardo ${ }^{2}$

FREITAS, Roberto Araujo de Moraes. MARTINS, Priscila Bernardo. La historia de la educación y la investigación científica en Brasil. Revista Científica Multidisciplinar Núcleo do Conhecimento. año 04, Ed. 12, Vol. 08, págs. 127-138. diciembre de 2019. ISSN: 2448-0959, Enlace de

acceso: https://www.nucleodoconhecimento.com.br/educacion-es/historia-de-laeducacion

\section{RESUMEN}

En el artículo actual, se abordan las transformaciones históricas de la actividad docente en Brasil, así como su participación en la investigación académico-científica, teniendo en cuenta desde las influencias derivadas de las religiones heredadas de Europa hasta las señas de identidad de los recursos tecnologías en manos de los usuarios nacionales. Este momento está marcado por la transición entre los siglos XX y XXI, lo que representa una revolución desenfrenada en el sector tecnológico, siendo Internet la principal herramienta de comunicación e investigación, que dio lugar a un largo proceso de adaptación. Es necesario mencionar la dificultad que hay en el

\footnotetext{
${ }_{1}^{1}$ Postgrado en enseñanza en educación superior, graduado en Ingeniería Informática.

2 Doctorado en Enseñanza de Ciencias. Máster en Enseñanza de La Ciencia. Especialización en Educación a Distancia: Preparación de materiales, tutoría y entornos virtuales. Especialización en Pedagogía Empresarial. Graduación en curso en Matemáticas. Graduación en Gestión de Recursos Humanos. Graduación en Pedagogía.
} 
proceso de aislar el conocimiento de su cultura local, opiniones y experiencias propias y el bagaje académico con el que un maestro necesita transmitir al cuerpo estudiantil, porque debe entender que el Brasil registra influencias de varios pueblos de todo el mundo, lo que nos ha hecho tener una cultura tan mixta. En medio de tantas transformaciones históricas, la actividad docente debe tener sus metodologías constantemente recicladas. Por esta razón, esta obra se vuelve más ardua ya que el profesor sufre del impacto de la red en el mundo, ya que se refleja en la productividad y fiabilidad de los resultados de las obras literarias y científicas. Esto siempre comprometerá la relación entre el conocimiento y la información.

Palabras clave: Tecnología, investigación, educación, fiabilidad.

\section{INTRODUCCIÓN}

El ejercicio de la enseñanza siempre ha sufrido cambios sociales, especialmente de acuerdo con los intereses ideológicos de cada región, ya sea por naturaleza religiosa o política. De la historia de Brasil, que data de 1500, la influencia del cristianismo en las primeras escuelas del país, orquestadas por la institución europea llamada companhia de Jesús, es relevante. A lo largo de los años, ha habido períodos de incentivos, así como descuido para los niveles de educación de la población. Esta brecha es una realidad que a día de hoy aún no se ha superado, principalmente porque es posible conferir una inversión muy baja en el sector académico, así como en la investigación científica, incluso si hay leyes que apoyan al sector, de alguna manera.

Ley que nació en Brasil Imperio, más específicamente en el gobierno de D. Pedro I. Lo anterior se llamaba la Ley de Directrices y Bases, una ley que ha estado experimentando, hasta el día de hoy, cambios en todos los gobiernos, siendo su versión más reciente fechada en 1996. El tradicionalismo educativo, dado por bibliotecas, libros, artículos, editoriales, etc., comienza a transformarse a finales del siglo XX con la aglomeración de información en Internet. Esto se refleja en la dificultad que tienen profesores e investigadores hasta el día de hoy en adaptarse a las nuevas 
tendencias de trabajo, ya que, en la misma medida en que facilitan el acceso a la información, reducen drásticamente la fiabilidad del contenido.

\section{LA HISTORIA DE LA EDUCACIÓN Y LA INVESTIGACIÓN CIENTÍFICA EN BRASIL}

\subsection{DESCUBRIMIENTO Y COLONIZACIÓN}

Los primeros indicadores significativos de la educación brasileña, en el sentido académico-escolar, están paralelos a la historia del país, desde su descubrimiento por el gobierno de Portugal, oficialmente en el año 1500. Desde siempre, el acceso a la información y la forma en que se crea y educa a las personas en Brasil tiene repercusiones en el modo en que se comporta la sociedad, porque se manipula de acuerdo con el deseo de la clase dominante. Según Ribeiro (1993), no era interesante para los colonos permitir el acceso a la información a los nativos, ya que era una estrategia capaz de provocar el impacto de culturas e intereses divergentes entre la corte y la población indígena.

La propuesta, a priori, era hacer que los individuos de la misa local colaboraran con el trabajo a ciegas y sumisamente y este objetivo fue diseñado por los europeos sobre la base de la distorsión de la identidad cultural y religiosa de la población brasileña nativa. Por esta razón, apenas tenían acceso a la alfabetización, y por lo tanto su cultura fue violentamente manipulada. Durante este proceso, varias naciones indígenas fueron diezmadas por no someterse al juicio jesuita. Desde Europa, los colonos contuvieron el apoyo de los jesuitas para difundir el cristianismo en Brasil como lo estaban haciendo en varios países a través de su institución matriz, la Compañía de Jesús. La organización ha erigido varias unidades educativas fundamentales en Brasil.

Veintiún años después de la llegada de los jesuitas, la Orden de la Compañía de Jesús ya podía tener cinco escuelas de educación primaria, en diversos lugares de todo el país, especialmente en las regiones costeras: Porto Seguro, Ilhéus, Espírito Santo, San Vicente y Sao Paulo de Piratininga. Con ellos, tres escuelas: Salvador, Río de 
Janeiro y Olinda (ALVES, 2009). Aquí, la diferencia entre la escuela y la escuela ocurre de acuerdo con el nivel de educación. Por lo tanto, lea una escuela para instituciones de educación primaria (Primer Grado o Escuela Primaria I y II, según la nomenclatura ldb/96) y una educación secundaria (Segundo Grado o Escuela Secundaria, según la nomenclatura establecida por LDB/96).

Los jesuitas fueron responsables de la educación en Brasil durante aproximadamente doscientos años. Primero, catequesis a los indios, a los que llamaban salvajes. Más tarde fundaron escuelas y misiones. Este proceso de difusión de las enseñanzas occidentales siguió hasta la expulsión de los jesuitas en 1759. Con esto, el proceso educativo entró en un período de estancamiento. Según Rosa (2014), la Orden de la Compañía de Jesús declinó después de aproximadamente 200 años de la llegada de los portugueses a Brasil. En ese momento, Sebastiáo José de Carvalho e Melo, el marqués de Pombal, era jefe de Estado y diplomático internacional de Portugal.

Incluso hubo un intento en el constituyente de 1823, según NISKIER (1969) debido a la proclamación de la independencia, para aumentar el sistema educativo, a través del artículo 250 que determinó la creación de escuelas primarias en cada aldea, gimnasios en cada distrito y universidades en ciertos lugares, pero esto fue sólo en teoría, porque el emperador disolvió el constituyente y al año siguiente dio la primera Constitución del Imperio y en el artículo 179 dijo: "La educación primaria es gratuita para todos los ciudadanos". En 1827, promulgó la Ley General que será la primera y única para la educación pública primaria hasta 1946. D. Pedro II, aunque se considera muy culto gobierna durante 49 años, pero sus proyectos en el sector educativo fueron tímidos y sin relevancia (ALVES, 2009, p. 10).

Como miembro de la élite portuguesa, le molestó mucho por el surgimiento del dominio social religioso de la Orden, ya que desequilibraba los poderes políticos del gobierno. Como resultado, el 22 de enero de 1759, se publicó la frase que determinó la exoneración y desnaturalización de jesuitas del territorio portugués (incluyendo Brasil). La situación sólo cambió con la llegada de la Familia Real. Durante el período de la 
monarquía, la educación siente los reflejos de la expulsión de los jesuitas. Don Pedro I ha gobernado durante años, pero no tiene noticias.

\subsection{INVERSIONES SOCIALES EN EL SECTOR EDUCATIVO Y LA INFLUENCIA DEL PROFESOR PAULO FREIRE}

Debido al largo período que duró la Ley General, el sector educativo en Brasil sólo sufrió importantes transformaciones a partir de 1946, después de la deposición de Getúlio Vargas, durante el período que se conoció como la Segunda República: cuando la dictadura de Vargas llegó a su fin y se reanudó el régimen republicano brasileño. En 1948, de acuerdo con lo que determinó la quinta constitución, se comenzó a trabajar en la Ley de Directrices y Bases, que fue promulgada en 1961. Simultáneamente con la promulgación del LDB, surgió el Método de Educación Popular del profesor Paulo Freire. El Movimiento Cultural Popular, MCP, guiado por el propio Paulo Freire, tuvo a los agricultores como los primeros estudiantes en pasar por esta experiencia, siendo alfabetizados de adentro hacia afuera, a través de su propio trabajo. Los resultados obtenidos -300 trabajadores alfabetizados en 45 días-impresionaron profundamente a la opinión pública.

Se decidió aplicar el método en todo el territorio nacional, pero esta vez fue apoyado por el Gobierno Federal. Ha habido un notable crecimiento en las inversiones en educación desde la promulgación del BID. La popularización de los métodos de enseñanza del profesor Paulo Freire inspiró a varias personas e instituciones, creando así nuevos proyectos y grupos sociales para apoyar la difusión de la formación y el conocimiento. Natal/RN fue la primera ciudad brasileña en aplicar esta didáctica con la Campaña de Alfabetización "Standing On The Ground Also Learns To Read", dirigida a adultos analfabetos. Alves (2009, p. 67) dice que también hay en ese momento "los Centros Populares de Cultura, vinculados a la Unión Nacional de Estudiantes; el Movimiento de Educación de base (vinculado a la Conferencia Nacional de Obispos de Brasil) y al Gobierno de la Unión."

En 1962, se creó el Consejo Federal de Educación, de conformidad con el artículo 9 de la Ley de Directrices y Bases. Esto se lleva en lugar de la Junta Nacional de 
Educación. En el mismo año, se crean los Consejos Estatales de Educación, el Plan Nacional de Educación y el Programa Nacional de Alfabetización, estos dos últimos por el Ministerio de Educación y Cultura, siguiendo la propuesta del Método Paulo Freire. Con la creación de este sistema, Freire inaugura una nueva etapa en la educación de adultos en Brasil. Este proyecto educativo, llevado a cabo por el Servicio de Extensión Cultural de la Universidad de Recife, bajo su liderazgo, llamado Sistema Educativo Paulo Freire, tuvo como objetivo principal el analfabetismo de adultos. Sin embargo, después de un corto período de tiempo de ejecución, estos proyectos fueron interrumpidos por el golpe militar de 1964. Así, en palabras de Oliveira (2001, p.56):

Hasta el golpe de estado del 31 de marzo de 1964, la relación entre los gobiernos progresistas y los movimientos populares centrados en la educación popular era colaborativa. Es decir, para hacer esfuerzos para implementar las propuestas de populismo y desarrollo brasileño. Algunos de los movimientos más expresivos de la educación y la cultura popular en Brasil nacen allí. Estas son expresiones de este movimiento: el Movimiento cultural popular (MCP), el Movimiento de Educación de Base (MEB), el Centro Popular de Cultura (CPC) y la Campaña "Standing on the Ground" también se aprende a leer". A partir de entonces, la educación y la cultura ya no se debieron a la simple formación del electorado. Se consideran los instrumentos de transformación de la estructura social, como espacios para la formación de seres conscientes, críticos y participantes.

Con el golpe militar de 1964, una fuerte represión recae en los movimientos sociales, que también surten efecto en los grupos e instituciones que trabajaron en proyectos de educación y cultura populares. El MEB, sin embargo, permaneció en funcionamiento en el Programa Nacional de Alfabetización. Entre 1970 y 1971, cuando Brasil, al establecer el acto institucional No. 05, en el gobierno de Costa Silva, entró en el período más represivo de la dictadura militar, un período llamado "golpe de Estado dentro del golpe". Durante este período, el gobierno optó por instalar una 
estructura aún más grande, el Movimiento Brasileño de Alfabetización - MOBRAL (Ley No 5. 379 de 15/12/67).

Entre sus objetivos, la difusión de la ideología oficial de desarrollo fue uno de sus objetivos, con miras a fortalecer el actual modelo de dominación y modernización. Sin embargo, en palabras de Bittar y Bittar (2012, p. 163), los gobiernos post-dictadura también fueron incapaces de resolver el problema y, además, "por no cumplir con la universalización de la escuela básica, una tarea llevada a cabo por la mayoría de los países paso del siglo XIX al XX, Brasil entró en el siglo XXI con esta herencia vergonzosa."

\subsection{INSTITUCIONES DE INCENTIVOS A LA INVESTIGACIÓN}

También a principios de la década de 1960, parte del gobierno llegó a ver una ventaja en la inversión en desarrollo tecnológico y científico. Durante este período, Fapesp Se creó la Fundación Estatal de Apoyo a la Investigación de Sao Paulo, y con ella varias otras instituciones relacionadas Brasil dentro. Entre los objetivos estaba la creación del modelo de posgrado strictu sensu. También es notable el SBPC Sociedad Brasileña para el Progreso de la Ciencia, creado en 1934, en la ciudad de Sao Paulo / SP, pero que comenzó a tener más visibilidad y autonomía en la transición entre los años 60 y 70. En 1988 Brasil promulgó su nueva constitución, conocida como la Constitución Ciudadana, cuyo artículo 208 establece que es el deber del Estado garantizar la escuela primaria obligatoria y gratuita.

[...] fue un hito histórico importante en la educación brasileña, ya que esta ley reestructuró la educación escolar, reformulando los diferentes niveles y modalidades de educación. [...]desencadenó un proceso de implementación de reformas, políticas y acciones educati[...]vas en lugar de frenar el proceso expansionista privado y restablecer las direcciones de la educación superior, contribuyó a exactamente lo contrario: se expandió e instituyó una diversificado y diferenciado, a través, sobre todo, de mecanismos de acceso, organización académica y cursos ofrecidos. En este contexto, creó los llamados cursos secuenciales y 
centros universitarios; estableció la figura de las universidades especializadas en el ámbito del conocimiento; implementaron Centros de Educación Tecnológica; reemplazó el vestibular por procesos selectivos; los planes de estudio mínimos y relajado los planes de estudio; creó cursos de tecnología e institutos de educación superior, entre otras enmiendas (BITTAR; BITTAR, 2012, pág. 165).

También vale la pena reiterar, unos años más tarde, las políticas adoptadas en ambos mandatos del gobierno del presidente Fernando Henrique Cardoso alentó, principalmente, el crecimiento de la matrícula en las escuelas públicas brasileñas. Era una política que tenía como objetivo atraer a más estudiantes desde la escuela primaria hasta la escuela secundaria. Por otra parte, el Estado dejó de priorizar, por lo tanto, la educación superior, lo que automáticamente dio lugar a la falta de recursos y también de incentivos. Esta situación negativa dio lugar, entonces, a la jubilación anticipada de los maestros, así como a una mayor incidencia en sus transferencias a sectores privados equivalentes. En este contexto, el gobierno comenzó la elaboración de una nueva versión para la Ley de Directrices y Bases de 1996.

\subsection{ENTRE ENCICLOPEDIAS ACADÉMICAS $Y$ VASTOS CONTENIDOS DE INTERNET PARA LA INVESTIGACIÓN CIENTÍFICA}

La investigación ha sido una práctica común en las escuelas desde la década de 1960, con la propuesta de transformar a los estudiantes en actores en su proceso educativo. Sin embargo, la investigación por sí sola no garantiza el desarrollo crítico de los estudiantes y depende de la práctica pedagógica guiada por el profesor. García (2017) afirma que el proceso de investigación se facilitó exponencialmente después de la domesticación de microcomputadoras e Internet. Pero esto significó algunos trastornos para la ciencia y el entorno escolar-académico: la descartedería de la información. Una gran cantidad de información no siempre es sinónimo de mucho conocimiento. Dado que la información se puede lograr en unos pocos clics, el interés en el aprendizaje ha disminuido. Hasta entonces, el ejercicio de hacer un trabajo 
escolar requería mucho más esfuerzo, ya que el estudiante debería dedicar unas horas en una biblioteca, buscando en numerosos libros la información deseada.

Para facilitar el proceso, hubo enciclopedias que presentaron varios temas, más tarde vendidos por números en quioscos. Actualmente, Internet ha sido la mayor fuente de datos para la investigación escolar. Esto comenzó a partir de la comercialización de microordenadores en entornos domésticos, que no pasaron mucho tiempo antes de tener acceso a la red (FERRARESI et al, 2009). Hasta entonces, las universidades y las instituciones escolares mantenían la cultura de fomentar la investigación de lo que estaba disponible en libros, enciclopedias, artículos científicos, etc. A día de hoy, los profesores se enfrentan a grandes dificultades para adaptar su trabajo a los recursos tecnológicos actuales, ya que Internet es un entorno en constante expansión y transformación. Dado que el número de usuarios cibernéticos crece exponencialmente en todo el planeta y teniendo en cuenta que todos disfrutan de la facilidad de producir y extraer contenido de la red, el uso de Internet como una fuente de investigación para el trabajo académico requiere mucho Cuidado.

Vale la pena señalar que el mismo fue creado, al principio, con fines académicos. El entorno académico-científico se refiere al grado de credibilidad dada la información extraída de la red. Debido a la inmediatez propuesta, vinculada a la nueva cultura de la comunicación instantánea electrónica, el porcentaje de estudiantes e investigadores que todavía hacen uso de libros de biblioteca, artículos impresos y similares para la escritura de sus tesis es mínimo. En cualquier caso, las universidades generalmente siguieron con la determinación de mantener sus bibliotecas actualizadas. Internet no alteró la credibilidad de los libros, por lo que estos siguen siendo los más apreciados en la búsqueda de información. Ferraresi et al (2009, p. 4) alegan que:

Las actividades de estudio y búsqueda a través de la comunicación en línea se llevan a cabo desde sitios de búsqueda, con un predominio del sitio de búsqueda Google (...). Y entre los sitios que los entrevistados conocen (...) universiabrasil, ibge y schoolar.google son más citados. 
En este nuevo momento, Ramos y Copolla (2009) resumen que el entorno académico debe estar a la vanguardia tecnológica, adaptando sus ambientes interiores a esta realidad. Internet se puede utilizar para diversos fines y los aprendices deben ser entrenados para obtener información concisa y confiable de la misma, dada su fragilidad, ya que cualquier usuario puede producir contenido. Se debe realizar un trabajo de análisis con el fin de verificar Internet como si se trata o no de una herramienta pedagógica, de acuerdo con los objetivos del usuario en un momento dado. Afirman que Internet al alcance del usuario doméstico permite romper las barreras del aula y acortar las distancias entre el profesor y el estudiante, si no puede o no quiere depender de un horario fijo, y por lo tanto la educación beneficia la colectividad.

(...) Internet (...) se ha convertido en una fuente de información potencialmente infinita y ha aportado diferentes tipos de ventajas $y$ nuevos problemas al profesor, porque al mismo tiempo permite intervenciones pedagógicas mucho más sofisticadas e incluso más interesantes que las clases tradicionales (...), llevan al maestro a enfrentar cambios en las relaciones de poder sobre el conocimiento: donde el maestro deja de tener control sobre el contenido de referencia y, comienza a administrar información impredecible (...). Por esta razón, para el uso de estas herramientas en la educación, no debe limitarse a la formación del profesorado como otra innovación tecnológica (RAMOS; COPOLLA, 2009, p. 7).

Alienta la capacidad de autoaprendizaje, pero no debe adoptarse como una panacea, como se había hecho con el libro de texto hasta entonces. Esto significa que, por mucho que los recursos tecnológicos se incorporen a la realidad académica, no pueden entenderse como la única herramienta de trabajo posible para la ejecución de actividades, sino que se traducen en dinamismo y creatividad, así como establece una conexión entre los conceptos teóricos y la vida privada de cada uno de los estudiantes. Internet es una fuente infinita de información y esto hace que el profesor pierda la 
imagen del titular de la información. Por lo tanto, es necesario tener cuidado al tratar con esta información.

\subsection{EL ROLE DEL PROFESOR EN LAS TRANSFORMACIONES METODOLOGICAS DEL AMBIENTE ACADEMICO}

Es indiscutible que la profesión docente se flexiona a medida que la estructura de toda la sociedad se vuelve en función de sus tradiciones culturales y recursos disponibles para el trabajo. El ser humano, en la modernidad, impulsa su trabajo para conectar a todos los sectores de la sociedad. Por esta razón, el buen maestro debe relajar su trabajo buscando la renovación constante de lo que tiene para ofrecer, con el objetivo de aumentar el rendimiento y la calidad del servicio, admitiendo que incluir nuevas herramientas a la realidad del aula puede traer aspectos Positivo. En este contexto, Ramos y Copolla (2000, p. 9), aluden, en su estudio, que:

El maestro adopta una nueva actitud. Aunque, una o otra vez, sigue desempeñando el papel de especialista que tiene conocimientos y/o experiencias que comunicar, más a menudo desempeñará el papel de asesor de las actividades del alumno, consultor, facilitador del aprendizaje de alguien que pueda colaborar para impulsar el aprendizaje de los estudiantes, desempeñará el papel de aquellos que trabajan en equipo, junto con el estudiante, buscando los mismos objetivos: una palabra, desarrollará el papel de mediación pedagógica.

La informatización de la enseñanza por sí sola no es suficiente para producir conocimiento. El maestro es, en primer lugar, un transmisor de conocimiento. Debe usar todo su equipaje académico para infundir interés en el estudiante para la investigación. Se sabe que Internet, cuando se utiliza correctamente, es una fuente de conocimiento. Sin embargo, cuando se usa indiscriminadamente y sin orientación, puede representar un flaco a la información. El trabajo académico en el que el estudiante utiliza sólo la red informática mundial como fuente de investigación para escribir su trabajo puede resultar en un trabajo inocuo. En este punto, notamos la 
importancia de la presencia del maestro, porque debe despertar, en el estudiante, la curiosidad de ir más allá de una simple lectura en Wikipedia.

El propio equipo docente del propio entorno académico desaprueba el uso de determinados sitios web para la aceptación de los ensayos de sus alumnos en sus artículos científicos, ya que la información, cuando se publica en Internet, se vuelve muy fácil de ser manipulada, facilidad con la que un usuario conectado también produce contenido. Por otro lado, este nuevo escenario trae consigo momentos de desequilibrio en su trabajo, ya que el estudiante ya no se atasca sólo al contenido que se le presenta, sino que también puede aparecer con preguntas distintas de la trama de la clase, basadas en el contenido disponible en Internet. Para Ramos y Copolla (2000), el profesor es un agente que debe buscar constantemente la actualización y reciclaje de su conocimiento de equipaje.

(...) la formación siempre debe considerar el contexto educativo en el que se inserta el profesor, por lo que lo incorpora a su dar a conocer y discutir el uso de estas tecnologías es necesario. Permitir que cada profesor, dentro de su realidad de formación y actuación, incorpore conscientemente estas herramientas y, no como un aparato tan simple, anime o ilustre sus clases (RAMOS; COPOLLA, 2000, p. 12).

Se sabe que las tecnologías implican procesos que se mejoran dinámica e ininterrumpidamente, haciendo que sus usuarios, y especialmente sus desarrolladores se involucren con el fin de mantenerse al día con las transformaciones que se han producido. En el entorno académico, en general, no es imprescindible que los profesores se preocupen por la arquitectura de desarrollo de los recursos tecnológicos, pero es interesante que sepa concatenar estos recursos a la realidad del aula para que los alumnos se sientan inmersos en sociedad de la información, incluso si se encuentran en el entorno académico-escolar. Sumergir la lección en una realidad virtual ofrece a los estudiantes una visión práctica de los temas abordados y los hace más críticos cuando se presentan correctamente. Si el profesor logra el objetivo de desarrollar la creatividad, la curiosidad y la interacción en los estudiantes, hará un mejor uso del contenido. 


\section{CONSIDERACIONES FINALES}

Presentando como punto de partida la historia de la educación en Brasil fue importante para delimitar las influencias de su gente en el interés de la investigación académica. Aunque legislada, la educación brasileña ha sufrido pocas inversiones en investigación y educación desde siempre y esto se debe a intereses políticos. El profesor Paulo Freire incluso trató de dejar su contribución y revertir la trágica imagen del analfabetismo en Brasil. Lo mismo fue referenciado internacionalmente y aplaudido por las grandes naciones y universidades, pero

Aquí el proyecto fue destruido y muy criticado. En el siglo XXI, el profesor ve dificultades para adaptarse a las nuevas tendencias de la sociedad con respecto a Internet, sustituir bibliotecas y editoriales y reducir la credibilidad de la autoría de la obra, ya que el acto de enseñanza no está directamente relacionado con la universo tecnológico, pero esto es lo que globaliza todos los sectores de la sociedad y en el trabajo.

\section{REFERENCIAS}

ALVES, W. L. U. A história da educação no Brasil: da Descoberta à Lei de Diretrizes e Bases de 1996. 2009. 76 f. Monografia (Especialização em Metodologia do Ensino Superior) - Centro Universitário Católico Salesiano Auxilium. Lins, 2009.

BITTAR, m; BITTAR, M. História da educação no Brasil: a escola pública no processo de democratização da sociedade. Acta Scientiarum Education, v. 34, n. 2, p. 157168, 2012.

FERRARESI, A. C. et al. Uso da internet como fonte de pesquisa entre universitários: um estudo de caso. In: XIII Encontro Latino Americano de Iniciação Científica e IX Encontro Latino Americano de Pós-Graduação - Universidade do Vale do Paraíba, 2009 
GARCIA, R. Antes do Google e da Wikipédia - as enciclopédias do passado. 2017. Disponível em: https://vejasp.abril.com.br/blog/memoria/antes-do-google-e-dawikipedia/. Acesso em: 17 dez. 2018.

OLIVEIRA, E. S. Diferentes sujeitos e novas abordagens da educação popular urbana. 2001. 152 f. Dissertação (Mestrado em Educação) - Universidade Federal Fluminense. Niterói, 2001.

RAMOS, M; COPOLLa, N. C. O uso do computador e da internet como ferramentas pedagógicas. PDE. 2009. Disponível em: http://www.diaadiaeducacao.pr.gov.br/portals/pde/arquivos/2551-8.pdf. Acesso em: 17 jan. 2019.

RIBEIRO, P. R. M. História da educação escolar no brasil: notas para uma reflexão. Paiadéia, n. 4, p. 15-30, 1993.

ROSA, T. F. lluminismo e a expulsão dos jesuítas do Império Português; as reformas pombalinas e o plano dos estudos menores. Revistas de História Regional, v. 19, n. 2, p. 361-383, 2014.

Enviado: Agosto, 2019.

Aprobado: Diciembre de 2019. 